\title{
Designing User Interfaces for Mobile Entertaining Devices with Cross-Cultural Considerations
}

\author{
Chien-Hsiung Chen ${ }^{1}$ and Chia-Ying Tsai ${ }^{2}$ \\ Graduate School of Design, National Taiwan University of Science and Technology \\ 43 Keelung Road, Section 4. Taipei, 106, Taiwan \\ ${ }^{1}$ cchen@mail.ntust.edu.tw, ${ }^{2}$ virginiatsai@gmail.com
}

\begin{abstract}
The purpose of this study is to explore the design process regarding how interaction designers in Taiwan deal with the OEM and ODM types of product and user interface design styles pertinent to mobile entertaining devices, such as MP3 players and portable media players (PMP). In addition to the discussion of what culture is and the way to design international user interfaces with cross-cultural considerations, detailed interaction design process with real world design examples is also introduced. It is hoped that the design process mentioned in this paper can be a good reference to interaction designers when they design product and user interface to satisfy users of various cultural backgrounds.
\end{abstract}

Keywords: Mobile entertaining device, Cross-cultural design, Interaction design, Usability testing.

\section{Introduction}

"The world is flat." As Friedman [2] points out that the physical boundaries among the world economic entities are disappearing. Designing products for international users all over the world will be the goal for future marketing strategies. Traditionally, the product industry in Taiwan has long been operated as the Original Equipment Manufacturing (OEM) and Own Designing \& Manufacturing (ODM) types of design styles. Only few corporate companies were able to create their product brand names and conduct their product design process as the Own Branding \& Manufacturing (OBM) style. In addition, due to the progress of advanced digital technology, the product lifecycle for mobile entertaining devices, such as MP3 players and portable media players (PMP), has been decreased because new products are introduced to the market daily. Many of these mobile entertaining devices were designed and manufactured in Taiwan based on the OEM and ODM types of product and user interface design styles for users of various cultural backgrounds. Therefore, the time required for product and user interface design and manufacture before it is released to the world market has been significantly reduced because of global competitions. The earlier a company introduces its innovative product to the world market, the more likely that this company may have a better chance to occupy a bigger portion of the product's market shares and may be able to lead the future development of this 
product. Therefore, it is important for an interaction designer to create an internationalized product and user interface with cross-cultural considerations to accommodate the majority of the international users' requirements.

The purpose of this study is to explore the design process regarding how interaction designers in Taiwan deal with the OEM and ODM types of product and user interface design styles pertinent to the mobile entertaining devices, such as the MP3 players and portable media players (PMP). Based on the summaries from interaction designers working for the major corporate companies designing and producing mobile entertaining devices in Taiwan, the design process pertinent to the OEM and ODM types of product and user interface design styles are discussed in this paper.

\section{What Is Culture?}

Culture can be viewed as "shared patterns of behavior" [5]. A cultural environment is be able to provide an individual with an emotional space in which set of beliefs, values, and behaviors can be commonly shared by all the members within the same society or ethnic group [1]. Cultural traditions (i.e., patterns) must be generally agreed upon by the majority of the members of the culture, not just by an individual alone. Therefore, within one culture, the majority of the members will share the same image perceptions pertaining to the value or even the interaction style of the mobile entertaining devices. However, if the same mobile entertaining devices are designed to be used among different cultures, more cross-cultural design considerations will need to be conducted to guarantee the product's success.

Vaske and Grantham [8] point out three basic characteristics of culture: (1) Culture is generally adaptive. It is generally adaptive to the particular conditions of both physical and social environments. (2) Culture is mostly integrated. It is mostly integrated in that the elements or features which make up the culture are mostly adjusted to or consistent with each other. (3) Culture is always changing. It is always changing because of adapting to certain cultural events or integrating with other cultures. An interaction designer should fully understand the characteristics of culture before $\mathrm{s}$ /he can design a product and user interface for users form different cultures.

Culture can also be viewed as communication [3]. That is, within one culture, all the members are able to interact with each other based on similar cultural behaviors. Hall [3] organized cultures by amount of information implied by the setting or context of the communication itself, regardless of the specific words spoken. He argued that cultures differ on a continuum ranging from high to low context. In high-context cultures, the communication is implied by a physical setting or by an individual's beliefs and values. Information is shared among all members of the same culture, but some have more privileged access than others. For example, Japanese, Mexican, and African-American cultures are all related to high-context cultures. In low-context cultures, the communication among culture members is expected to be explicit, and everyone has equal access to available information. Examples of low-context cultures include German, Swedish, and European-American cultures. In the context of crosscultural design, the communication between a human and a product or a user interface is moving from low-context to high-context interaction. This can be due to that fact that the progress of digital computing technology has made the traditional rigid 
control on a product or a user interface no longer necessary. Instead, intelligent microchips in the $21^{\text {st }}$ century have enabled multiple and flexible interaction styles to facilitate the user's interactions. That is, the intelligent product is able to sense the user's task intentions and automatically execute the functions for the user.

\section{Designing International User Interfaces with Cross-Cultural Considerations}

To an interaction designer, the purpose of conducting international user interface design is to create useful and effective user interfaces which can be utilized by all the potential users with various cultural backgrounds. In fact, international user interface design should be considered as a cross-cultural collaborative work between interaction designers and users from different cultures [4]. Designing international user interfaces requires taking the concept of both internationalization and localization of user interfaces into account. Internationalization is the process of designing a base user interface which can be further integrated with various cultural factors to meet with different cultural needs. Localization is the process of adapting an internationalized user interface based on the features of a particular culture. In fact, the process of interface internationalization will facilitate the process of interface localization as well. The process of interface internationalization can provide a dynamic framework (i.e., the structure) in which interface localization can be implemented by adding cultural factors into the design. Because the internationalization of user interfaces requires intensive cross-cultural design considerations, an interaction designer will need to identify and separate basic principles regarding user interface design into culturally independent and culturally dependent variables. The culturally independent variables are the variables used to help interface internationalization, and the culturally dependent variables can be used to facilitate interface localization.

\section{The OED and ODM Design Process in Taiwan}

Because of the OEM and ODM types of product and user interface design styles in Taiwan, most of the product and user interface design projects require interaction designers to complete the design process within three to six months. Otherwise, the proposed products may not be able to occupy a vital place in the international market. Therefore, interaction designers working on this type of design project will need to construct a unique design process to ensure the deadline can be met. This unique design process may include seven stages described as follows:

\subsection{Understand the Design Goal}

Because of the time constraint, the interaction designer will need to fully understand the goal of the product or the user interface design in order to generate suitable design concepts. To do this, the interaction designer needs to understand three design issues, i.e., the user of the product, the function of the product, and the use environment of the product. 
The User of the Product. When conducting the OEM and ODM design project, an interaction designer first needs to know who the target users are. This is because different user groups may have different physical and psychological requirements towards that product or user interface. Their perceptions on the graphical user interface (e.g., icons and menu designs) and interaction styles on the solid user interface (e.g., buttons and switches) may be different as well. In addition, target users' general characteristics are also important for the design considerations. For example, the color used on the display heading of a mobile entertaining device may adopt the "matured colors" style if it is designed for the middle-aged business users (see Fig. 1). On the contrary, if the mobile entertaining device is designed specifically for teenagers, "vivid colors" tend to be used on the display (see Fig. 2).

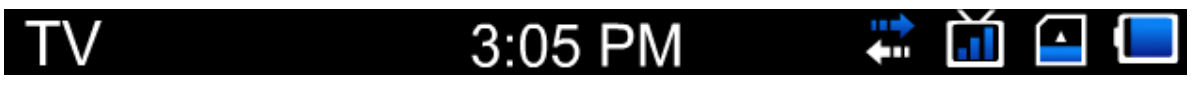

Fig. 1. Matured colors are adopted for middle-aged business users

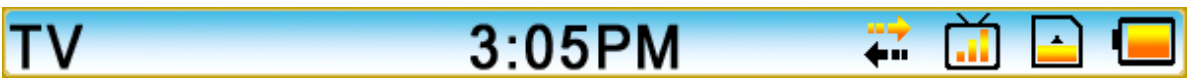

Fig. 2. Vivid colors are used for teenagers

The Functions of the Product. The functions provided in the mobile entertaining devices will affect how users interact with the user interface. For instance, the function of global positioning system (GPS) is often incorporated within this type of mobile entertaining device. In addition to provide users with precise position information, other design factors, such as battery capacity, fall protection, and water protection, are also important and an interaction designer needs to take these design factors into serious account.

The Use Environment of the Product. Depending on the user's work environment, an interaction designer will need to consider if this mobile entertaining device will be designed by adopting touch pen interaction style or just allow users to interact with the interface by using their finger tips. If it is designed for touch pen interaction, more function icons and detailed operation icons can be provided on the display because users can use a touch pen to conduct a more precise interaction (see Fig. 3). On the other hand, if this device allows user to interact with its user interface by using their finger tips, less function icons should be provided on the display and the size of the function icons should be larger for easier interactions (see Fig. 4).

\subsection{Plan for Systematic and Series Designs}

Because of the OEM and ODM characteristics, the product development cycle and lifecycle are very limited in Taiwan. To an interaction designer, the design goal is to put the product into the market as early as possible without sacrificing its quality. Once the product is in the market for around three to six months, a new generation of that similar product will replace its market position. Because this new generation is not very much different from its predecessor and only slightly changes have been 
made, an interaction designer will need to plan this type of design strategies in advance in order to create new products based on systematic and series considerations. By so doing, the interaction designer not only can minimize the production cost, but also can control the time frame for designing a new product. For example, Fig. 5 is the display showing the original functions of a mobile entertaining device. Fig. 6 is the new design illustrating more functions than its predecessor. The interaction designer should be able to complete designing the new generation within the possible shortest time.
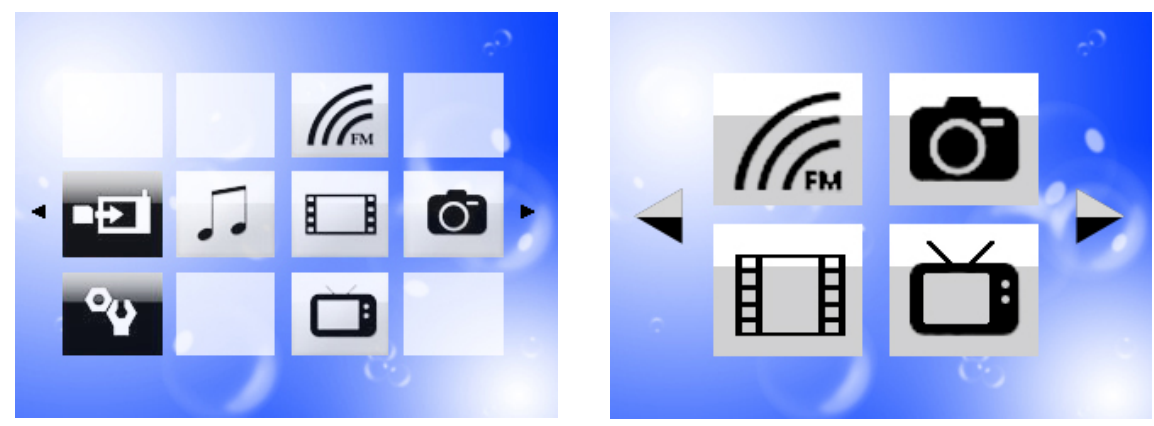

Fig. 3. The display with smaller icons de- Fig. 4. The display with larger icons designed signed for touch pen interaction for finger tips interaction

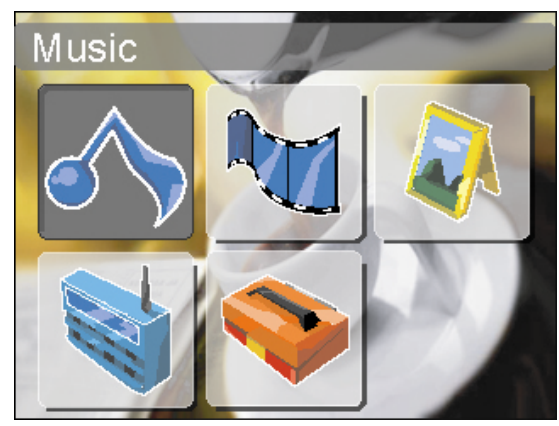

Fig. 5. The original design showing the functions of a mobile entertaining device

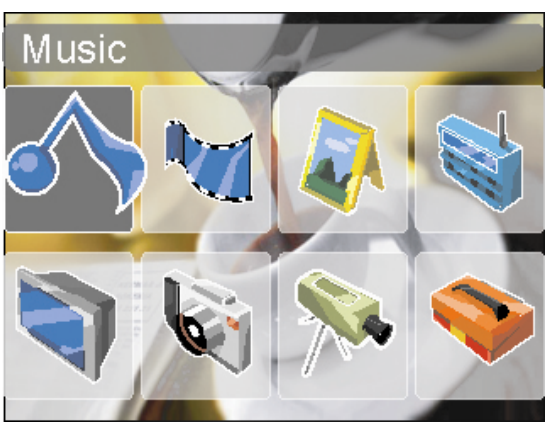

Fig. 6. The new design illustrating more functions than its predecessor

\subsection{Communicate Well with Other Design Teams}

The product and user interface design process in the current OEM and ODM design industry tends to be a team work. That is, an interaction designer cannot complete the design by himself/herself. That is, s/he needs to work with project manager, electrical engineer, mechanical engineer, software engineer, and other stakeholders. In order to complete the design within the scheduled time, all the parties need to communicate well. Knowing other team members' requirements in advance will also ensure the quality of the design. For example, when an electrical engineer is testing the display quality of a mobile entertaining device, the interaction designer may provide him/her 
with various styles of icon designs to be shown on the display (e.g., straight and curve borders, black and white, gray, and full colors, and degree of complexity). Fig. 7 demonstrates the result of an icon shown on an 8-bit display which can present 256 colors. The gradation quality is much better than that on a 4-bit display illustrated in Fig. 8. This is because different display quality may be equipped with different resolutions and limitations as related to price differences. What the engineer wants to achieve is to find out the combination of best presentation quality and lowest display cost to help win the product's price competition.

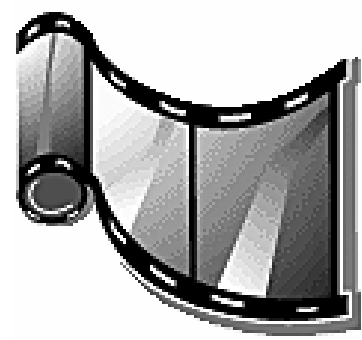

Fig. 7. An icon shown on an 8-bit display Fig. 8. An icon shown on a 4-bit display prepresenting 256 colors

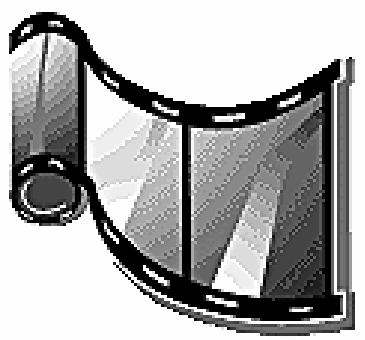

senting only 16 colors

\subsection{Conduct User Interface Design}

Once an interaction designer understands the client's design specifications and the user's requirements, s/he will start conducting the user interface design. It is an iterative design process emphasizing on the design and testing of generated ideas. During this stage, three types of design variations should be kept in the interaction designer's mind, i.e., hardware variation, content variation, and structure variation, to help achieve best design quality. Detailed explanations are provided below.

Hardware Variation. The interaction designer should always keep in mind that the product and user interface that $\mathrm{s} /$ he is currently working on may just exist in the market for a short period of time. Therefore, it is very important to prepare the new generation of the product and user interface in advance. Sometimes the new generation may be different from its predecessor in hardware requirements. For example, the original mobile entertaining device may be designed to be used on a 3.5" display (see Fig. 9). Nonetheless, the new generation may be used on a 7" display. The interaction designer should maintain the user interface design flexibility so the original design can be easily modified within the possible shortest time with the same display quality (see Fig. 10).

Content Variation. The design strategy for the OEM and ODM types of product and user interface design styles is to constantly provide new products on the market to attract users' attentions and, at the same time, encourage them to purchase these new products. Therefore, an interaction designer needs to be aware that the new generation may just have minor changes to its predecessor in order to save the cost of 
developing a whole new product or user interface. Most of the time, the minor change may just be the addition of a new function to the existing product and/or fixing the current problems. If the software of the user interface is designed with objectoriented considerations, it will not be too difficult for a software engineer to modify the coding of the existing software.

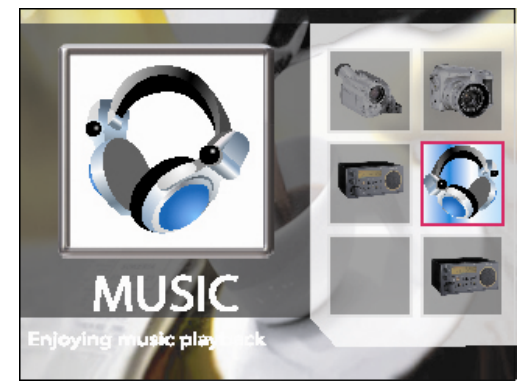

Fig. 9. The mobile entertaining device Fig. 10. The mobile entertaining device designed with designed with a 3.5 " display

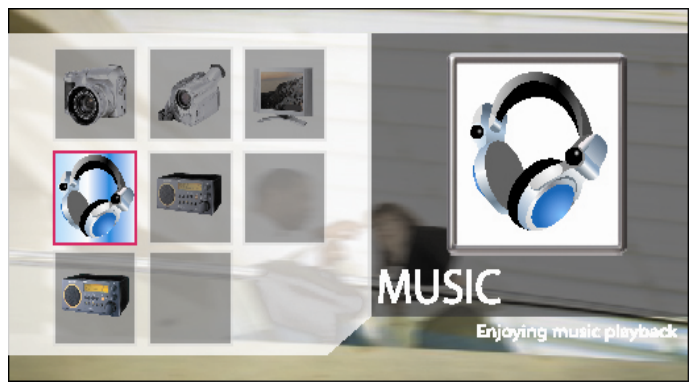

a 7" display

Structure Variation. The process of modifying the structure of a user interface can be very complicated and sometimes very difficult. Very often, the interaction designer may need to start the design process all over again. For example, Fig. 11 shows that the function icons on the main menu of a mobile entertaining device can be rotated and controlled by two arrow buttons on the sides. Fig. 12 illustrates that the function icons on the main menu can be chosen by touch-sensitive control style. Though these two user interfaces look similar, they are created based on two different interaction styles. It will be very difficult to convert one design based on the other. Therefore, it is important to obtain users' viewpoints in advance and inform the client which design can best satisfy most uses' interaction styles as soon as possible. Once the design decision has been made, try not to modify the design again for it can be very time and resources consuming.
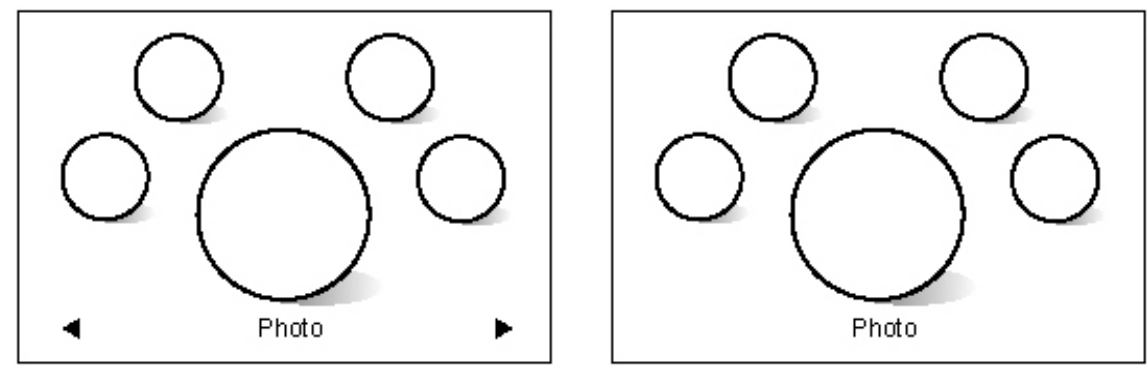

Fig. 11. The functions on the main menu can Fig. 12. The functions on the main menu can be rotated and controlled by two arrow be chosen by touch-sensitive control style butons 


\subsection{Implement Cross-Cultural Design Considerations}

There are two design strategies to be used before the product is put into the market, i.e., design for the general public and design for a specific target user group. These two user groups may have their own cultural characteristics. Very often, the strategy of designing for the general public will be adopted when the product is first introduced to the market. The purpose is to draw the public's attention and by so doing can also promote the product's brand name image. After that, limited editions with minor modifications (i.e., textures, colors, or endorsements from a famous person) of the same product will be introduced to the market to prolong the product's lifecycle. In order to conduct the second strategy, an interaction designer should be very aware of the target users' cultural features so that the limited edition can attract their attentions. Furthermore, in order to achieve the goal of conducting product variation, the concept of module design will be considered beforehand. That is, the changeable product or user interface elements should be designed in the forms of flexible modules. Therefore, the new generation of the product can be modified with less efforts and costs but still can be designed with a fresh new look. For instance, Fig. 13 shows the icon designs using black and white colors and simply style that can be used on a less expensive mono-colored display. Fig. 14 illustrates the icons designed by adopting more complex lifestyle images. This type of design is often used in the Asian market because of cultural characteristics. Fig. 15 demonstrates the icons designed by adopting the image of glassware in Chinese culture to help promote the quality of the product and user interface.

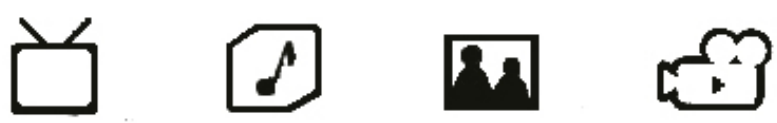

Fig. 13. The icons designed with black and white colors and simple style to be used on a monocolored display

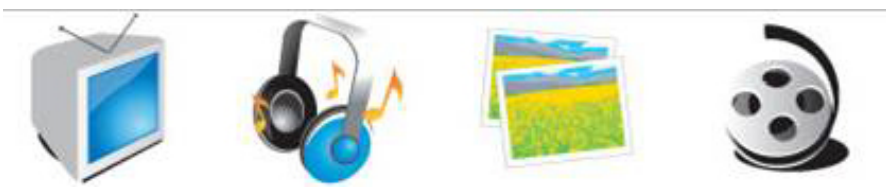

Fig. 14. The icons designed by adopting more complex lifestyle images and are often used in the Asian market

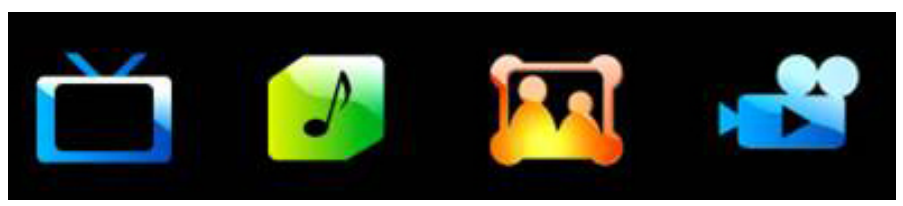

Fig. 15. The icons designed by adopting the image of glassware in Chinese culture to help promote the quality of the product and user interface 


\subsection{Construct User Interface Prototypes}

Because of the OEM and ODM characteristics, up to now, the interaction designer has spent a lot of time in designing the product and user interface. The time tends to be running out and s/he may not have enough time to construct the user interface prototype for testing purpose. The interaction designer may just spend one day to ask his/her colleagues or someone else working in a nearby office to act as a user to help provide opinions. After a brief modification, the interaction designer may transfer the user interface design to the software engineer for coding process. It is very likely that the user interface design may still contain potential interaction problems. The interaction designer and software engineer will need to jointly solve these unfound problems along the coding process. Nonetheless, if there is time for constructing user interface prototypes, two types of prototypes can be made during the design process, i.e., low-fidelity prototype and high-fidelity prototype. Fig. 16 shows the low-fidelity prototype to be used for design discussions. Fig. 17 illustrates high-fidelity computer simulation prototype to be used for usability testing to help acquire information regarding user preference and performance.

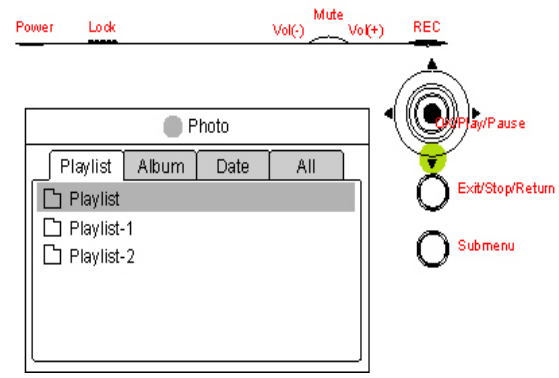

Fig. 16. Low-fidelity prototype used for design Fig. 17. High-fidelity computer simulation protodiscussions

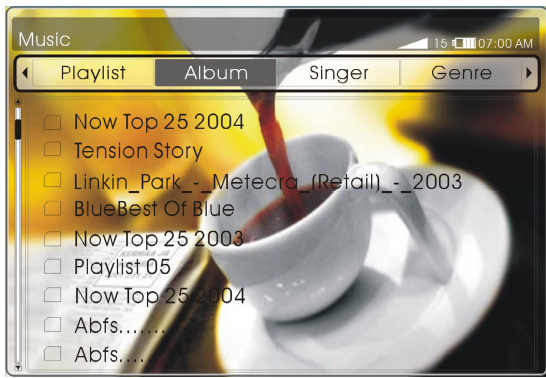

type used for usability testing

\subsection{Conduct Interface Usability Testing}

The International Standards Organization (ISO) defines usability as the effectiveness, efficiency, and satisfaction with which specified users can achieve specified goals in particular environments (ISO DIS 92411-11). Usability testing can be conducted by means of an interface prototype to assess the usefulness of an actual design. The overall goal of usability testing is to identify usability deficiencies existing in the proposed design before its release. The intention is to ensure that the new design will be very easy to learn and use, and that it can provide various functions valued by a target user group or users with various cultural backgrounds.

Under an ideal situation, the process of testing the product and user interface usability should be conducted in the target users' cultural environment in order to obtain the first hand information. However, in Taiwan, because of the time and resources constraint, the interaction designer may not be able to conduct a full scale usability testing by recruiting real users from oversea. Not to mention that $\mathrm{s} / \mathrm{he}$ may need lots of time to conduct the experiment and perform data analysis. Therefore, most of the 
time, the technique of heuristic evaluation will be conducted at the interaction designer's company. That is, the interaction designer may invite 3 to 5 expert users with different design backgrounds (e.g., product design, Website design, graphic design, etc.) to take part in the usability testing process. These experts may spend 1 to 3 days playing with the product and the user interface. According to Nielsen [6][7], most of the major design problems can be identified by these experts. After that, the interaction designer may still have some time to co-work with the software engineer for the last stage modifications before the product is released to the market.

\section{Conclusion}

Because of the OEM and ODM types of product and user interface design styles in Taiwan, the time required for the design development is strongly constrained. In order to compete and survive in the international market, an interaction designer will need to construct his/her own unique design process to fit in this rapidly changing environment. This research study demonstrates the unique product and user interface design process based on cross-cultural considerations with real world design examples. It is hoped that this unique design process can be a good reference for interaction designers to help design product and user interface that can satisfy users of various cultural backgrounds.

Acknowledgments. Financial support of the research by National Science Council under the grant NSC 95-2221-E-011-046 is gratefully acknowledged.

\section{References}

1. Ember, C.R., Ember, M.: Anthropology. Prentice-Hall, Englewood Cliffs, NJ (1977)

2. Friedman, T.L.: The World is Flat: A Brief History of the Twenty-First Century. Farrar, Straus and Giroux, New York (2005)

3. Hall, E.T.: The Hidden Dimension. Doubleday, New York (1969)

4. Ito, M., Nakakoji, K.: Impact of Culture on User Interface Design. In: del Galdo, E.M., Nielsen, J. (eds.) International User Interfaces, John Wiley \& Sons, New York (1996)

5. Mead, M.: Coming of Age in Samoa. Modern Library, New York (1953)

6. Nielsen, J.: Usability Engineering at a Discount. In: Salvendy, G., Smith, M.J. (eds.) Designing and Using Human-Computer Interface and Knowledge Based Systems, Elsevier, Amsterdam (1989)

7. Nielsen, J.: Big Paybacks from 'Discount' Usability Engineering. IEEE Software 7(3), 107-108 (1990)

8. Vaske, J.J., Grantham, C.E.: Socializing the Human-Computer Environment. Ablex, Norwood, NJ (1990) 